\title{
Load- and Interference-Aware Channel Assignment for Dual-Radio Mesh Backhauls
}

\author{
${ }^{\dagger}$ Michelle X. Gong, ${ }^{\ddagger}$ Shiwen Mao and ${ }^{*}$ Scott F. Midkiff \\ ${ }^{\dagger}$ Networking Technology Lab, Intel Corporation, Santa Clara, CA 95054 \\ ${ }^{\ddagger}$ Dept. of Electrical and Computer Engineering, Auburn University, Auburn, AL 36849-5201 \\ *The Bradley Dept. of Electrical and Computer Engineering, Virginia Tech, Blacksburg, VA 24061
}

\begin{abstract}
The combination of multi-radio nodes in conjunction with a suitably structured mesh architecture has the potential to solve some of the key limitations of present day mesh networks. We propose and evaluate two practical and self-stabilizing channel assignment algorithms for multi-channel dual-radio mesh backhauls. The objective is to find a channel assignment that maximizes network capacity through balancing traffic load and/or co-channel interference on available channels. Through simulations, we show that the proposed scheme can effectively improve the capacity of wireless mesh networks.
\end{abstract}

\section{INTRODUCTION}

In a multi-hop wireless mesh network that consists of single radio mesh access points (MAPs), throughput decreases approximately between linearly and exponentially with an increase in hop count due to interference generated by neighboring MAPs on the same channel. In fact, it has been proven that the end-to-end throughput available to each node in the same network is $O(1 / \sqrt{n})$, where $n$ is the number of nodes in the network [1]. Specifically, due to contention from subsequent forwarding nodes, the end-to-end throughput that a daisy-chain network can achieve is only about $1 / 7$ of the nominal link bandwidth [2]. Because utilizing different channels on adjacent radio links divides one big collision domain on the same channel into smaller collision domains on different channels and, thus, facilitates parallel packet transmission, a multi-channel mesh backhaul is a promising approach to boost the overall network throughput.

Currently, many mesh networks utilize only one out of the 24 available channels in the $5 \mathrm{GHz}$ band for their backhaul transmission. Since the backhaul capacity limits the total capacity that can be achieved by the network, it is critical to improve the capacity of mesh backhaul and to improve quality of service (QoS) provisioning by using multiple channels. Some prior work considers routing and channel assignment jointly [3], [4]. Ideally, an optimal solution should consider all aspects of radio resource management. Yet, achieving optimality for a joint solution is extremely difficult given the fact that optimizing one resource type can impact the management of other resources. A channel selection algorithm designed for rapidly-changing network status might be able to adapt to the dynamics of network conditions. However, such an approach would frequently change the connectivity between different mesh nodes, not only affecting the stability of the mesh backhaul, but also adversely impacting the end-to-end network performance.

In this paper, we propose to decompose the resource allocation problem into two parts: i) routing and ii) channel assignment, which are independently updated and optimized. Further, we assume that channel assignment does not alter the network topology (i.e., as a constraint for channel assignment). In addition to reducing the complexity of resource allocation algorithms, there are two practical reasons why we decompose routing and channel assignment. First, route changes cause packet losses and packet reordering, which significantly degrades TCP performance. Second, in a municipal mesh deployment, mesh backhauls need to operate at high data rates. Because of the complex radio propagation environments in most cities, few mesh deployments have rich link connectivity. Most nodes in a network may experience interference either internally or externally, but do not have alternative routes that provide high data rates. As a result, channel assignment is likely to be a better choice than dynamic routing for providing performance gains in terms of load balancing and interference mitigation. Dynamic routing is also widely used in municipal mesh products, but only as a means for self-healing and resilience.

This paper addresses the dynamic channel assignment problem of municipal mesh networks. We consider the optimization problem of channel assignment by taking into account factors such as the distribution of traffic load over the mesh network and interference generated from external networks. We develop two practical channel assignment algorithms for multichannel dual-radio mesh backhauls. Computed on a central controller, the algorithms intelligently select channels for mesh nodes based on the chosen metrics. Specifically, Algorithm 1 exploits the tree structure of wireless mesh backhauls for estimating the traffic load at each mesh point (MP) and seeks to balance traffic load and minimize interference within the mesh network. Based on actual network measurements, such as average traffic load and external interference, Algorithm 2 not only minimizes interference within the network, but also minimizes interference between the mesh network and other collocated wireless networks. Through ns 2 simulations, we show that the proposed channel assignment algorithms can improve the network capacity by up to three fold.

The remainder of the paper is organized as follows. Section II presents the channel assignment problem. The proposed algorithms are presented in Section III. Our simulation eval- 
uations are presented in Section IV. Section V summarizes related work and Section VI concludes the paper.

\section{Problem Statement and Assumptions}

\section{A. Problem Formulation}

Many channel assignment problems can be formulated as graph coloring problems with different constraints. A wireless mesh network can be represented as an undirected graph $G=$ $\{V, E\}$, where $V$ represents the set of mesh nodes and $E$ denotes the set of edges that represent radio links.

Let $C$ be the set of available channels. If the number of available channels, the cardinality of $C,|C|$, is sufficiently large, the channel assignment algorithm should assign distinct channels to every node within a collision domain. However, in many cases, depending on the signal-to-interference ratio (SIR) required at the receivers, the number of available channels may be less than the number of nodes in a collision domain. Thus, channels have to be carefully assigned to mesh nodes to avoid channel conflicts as much as possible.

Typically there can be many optimality criteria for channel assignment. The optimality criterion we choose for the proposed channel assignment schemes is to minimize the maximum traffic load as measured by percentage of medium utilization on each channel within any collision domain. The rationale for this choice is explained below. Most wireless mesh networks are based on the IEEE 802.11 (or Wi-Fi) standards, which utilize carrier sense multiple access (CSMA). Because all 802.11 devices on the same channel within the same vicinity share the medium, the lower the load on the medium by other nodes, the more medium capacity is available to the node of interest. Thus, medium utilization is a direct indicator of traffic load and interference on the channel. Moreover, since IEEE 802.11 data packets are typically sent at different data rates, medium utilization is a better metric than average throughput.

\section{B. Network Model and Assumptions}

We consider an $N$-node wireless mesh network in which all nodes are stationary. Each node is a mesh point (or, mesh router). A typical mesh architecture is illustrated in Fig. 1. A mesh point that also connects to external networks is called a mesh point portal (MPP), while a mesh point that supports associated wireless clients is called a mesh access point (MAP). Because most traffic carried through municipal mesh networks is to and from the Internet, after the routing algorithm converges, a routing tree that is rooted at the MPP will be formed regardless of the routing protocol adopted, where each MP or MAP can choose a single best upstream node and one or more downstream nodes.

We assume all nodes are equipped with dual backhaul radios. Even though, theoretically, a mesh node can be equipped with any number of radios, it is challenging and cost prohibitive to manufacture a mesh node with more than two radios operating in the same band. To improve network performance and to ensure traffic separation, the $2.4 \mathrm{GHz}$ band is usually used for client access whereas the $5 \mathrm{GHz}$ band is often used for

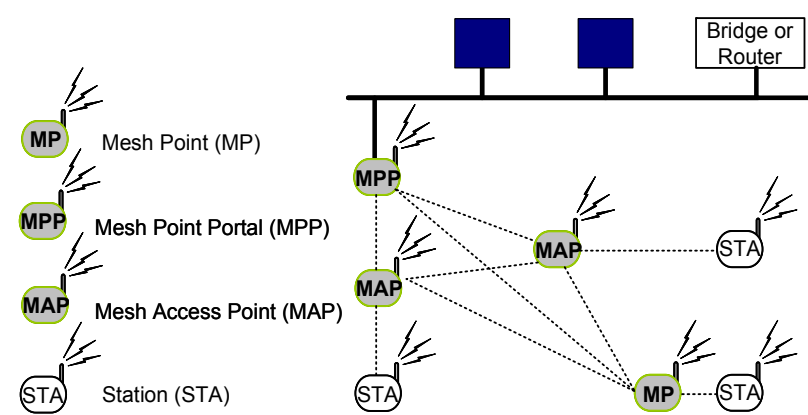

Fig. 1. Mesh architecture defined in IEEE 802.11s.

carrying backhaul traffic. Throughout this paper, we assume all mesh nodes are equipped with two $5 \mathrm{GHz}$ backhaul radios and a $2.4 \mathrm{GHz}$ client radio. Leading mesh providers, such as Cisco and Strix, have products supporting such an architecture.

We further constrain that a node does not frequently switch channels to communicate with different neighboring nodes. Instead, a node maintains an upstream radio that communicates with its upstream node and a downstream radio that communicate with its downstream nodes. There are two reasons for this choice: i) to avoid overhead of frequent channel switching, and ii) to avoid the negative impact of channel switching on other IEEE 802.11 users using the same channel, as discussed below.

First, changing from one channel to another incurs a nonnegligible channel switch delay, which ranges from $500 \mu s$ to a few $m s$, depending on implementation. Because each MAP typically needs to communicate with more than two neighbors, if neighbors are assigned different channels, frequent channel switches become necessary to maintain peer links with multiple neighbors. This type of frequent channel switch has a high overhead, which reduces available network capacity.

Furthermore, when one node switches to a new channel, it may have missed the preamble of an on-going packet transmission. According to the IEEE 802.11 standard, the carrier sensing threshold for receiving an IEEE 802.11a packet is $-82 \mathrm{dBm}$, whereas the carrier sensing threshold for non802.11 interference is $-62 \mathrm{dBm}$. If the preamble of a packet is missed, the receiver would not recognize that it is a packet and, thus, the carrier sensing threshold becomes $-62 \mathrm{dBm}$ instead of $-82 \mathrm{dBm}$. Because of the $20 \mathrm{dBm}$ difference in two thresholds, the node may sense the medium as idle and start its own transmission, which causes a collision. For instance, if the received signal strength indicator (RSSI) shows $-80 \mathrm{dBm}$ when the preamble of the packet is received, the receiver recognizes that this is an IEEE 802.11a packet and applies the $-82 \mathrm{dBm}$ threshold. Because $-80 \mathrm{dBm}$ is higher than $-82 \mathrm{dBm}$, the receiver asserts that medium is busy and refrains from transmitting. However, if the preamble is missed, the receiver does not recognize that it is an IEEE 802.11 packet. Utilizing the $-62 \mathrm{dBm}$ threshold for non-802.11 interference, the node asserts that the medium is idle and starts its own transmission, which may collide with the on-going transmission.

Because channel switching causes varying connectivity in 
the mesh backhaul, incurs high overhead, and may cause unnecessary packet collisions, we assert that frequent channel switching should be avoided as much as possible. In fact, in real mesh deployments, channels are often reassigned on an hourly or daily basis.

\section{Channel Assignment Algorithms}

In a wireless mesh network, the primary design goal of the channel assignment algorithms is load balancing, i.e., to minimize the maximum traffic load on any channel that is chosen by a designated node within this node's collision domain [3]. A collision domain is defined as a node's $(k+1)$ hop neighborhood, where $k$ is the ratio between the carrier sense range and the communication range. The carrier sense range can be approximately determined by the transmit power, the path loss, and the receiver sensitivity at the lowest data rate, whereas the communication range is determined by the transmit power, the path loss, and the receiver sensitivity at the desired data rate. In the case of a wireless mesh network operating in a suburban environment, $k$ is approximately 2 , which means the carrier sense range is about twice the communication range.

Given the characteristics of the mesh backhaul, we need two additional constraints for channel assignment. First, because each node is equipped with two backhaul radios, each node is assigned two unique channels, one for the upstream communications with its parent node and the other for downstream communications with its children. All children of the same parent share the same upstream channel to avoid channel switching overhead at the parent node and to avoid topology changes after a channel switch. Second, traffic aggregates in mesh networks. The closer a node is to the MPP or root AP (RAP), the heavier the traffic. Thus, a node with a lower hop count to the MPP must be given higher priority than a node with a higher hop count when assigning channels. With these considerations, we develop two channel assignment algorithms for the dual-radio mesh backhaul.

\section{A. Channel Assignment Exploiting Mesh Network Structure}

The first channel assignment algorithm, called Algorithm 1, exploits the tree structure of mesh backhauls. In Algorithm 1 , the traffic load at each MP is estimated and used. The goal, termed Metric 1, is to minimize the maximum number of weighted nodes sharing the same channel within the a collision domain, i.e.,

$$
\text { Minimize over } c_{j} \in C: \max \sum_{i=1}^{N_{j}} \omega_{i},
$$

where $N_{j}$ is the number of nodes in the same collision domain that use the same channel $c_{j} \in C$ to communicate with its parent; and $\omega_{i}$ is the weight assigned to the $i$ th node and it is calculated as the number of nodes in the subtree rooted at the $i$ th node. The purpose of this weight is to consider the traffic aggregation factor. Since in a tree structure, a node needs to carry all traffic from its children, as well as its own traffic, $\omega_{i}$

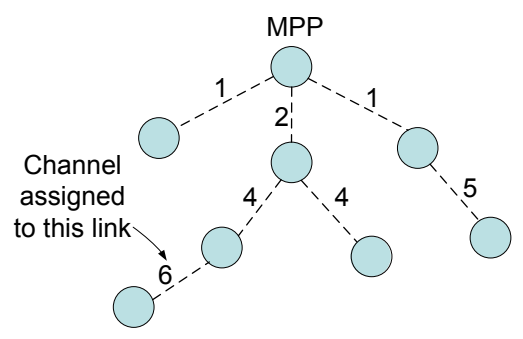

Fig. 2. A channel assignment example for a mesh backhaul.

can serve as an indication of the potential traffic load at mesh node $i$.

Fig. 2 shows an example of RF channel assignments for a mesh backhaul. Recall that each MP is equipped with two radios. The MPP uses both radios for communication with its downstream MPs. To avoid high channel switching overhead and to balance traffic, all other MPs use one radio for upstream communication and the other radio for downstream communication.

Although the optimization problem in (1) can be solved using integer programming techniques, we develop a greedy algorithm to obtain suboptimal solutions at low complexity, as shown in Fig. 3. To ensure fast routing convergence at the bootstrap phase and to facilitate fast recovery from major disruptions, initially all mesh APs including the MPP are on the same (bootstrap) channel. ${ }^{1}$ As shown in Fig. 3, the outer loop of Algorithm 1 is a centralized breadth first search algorithm, which leverages knowledge of the routing tree structure and operates at the network controller. During the tree formation phase, the network controller compiles a topology of the backhaul when mesh nodes register with the controller and report their hop counts and upstream and downstream nodes. The breadth first algorithm examines mesh nodes hop by hop, starting from the first hop and incrementing the hop count by one for each iteration of the loop. The algorithm stops after exhausting all hops. This breadth first algorithm is adopted to meet the second constraint, i.e. giving higher priority to nodes with lower hop counts. Note that Algorithm 1 starts only after the convergence of the routing processes, so as to avoid instability when both protocols try to optimize the network performance simultaneously. The inner loop in Fig. 3 examines the MAP with the worst cost at every iteration. A mini-max algorithm is applied where the goal is to minimize the maximum value of the proposed metric. At each iteration, the algorithm tries to optimize the overall cost by assigning a channel from the available channel set to minimize the cost of the MAP with the previously worst metric.

More specifically, Algorithm 1 operates as follows.

1) It first assigns two channels to the MPP. The MPP will use both channels to communicate with its children since it has no parent. The algorithm divides the children of

\footnotetext{
${ }^{1}$ Note that a bootstrap channel is not a common control channel as described in the literature (e.g., in [5]). A bootstrap channel is only used during the initial network setup phase, whereas a common control channel is used throughout the duration of the network operation.
} 
Let $\mathrm{V}=\{\mathrm{v} \mid \mathrm{v} \neq$ Root and $\mathrm{v} \in \mathrm{V}\}$

Let $\mathrm{Q}=\{\mathrm{c} \mid \mathrm{c}$ not taken by root MP and $\mathrm{c} \in \mathrm{C}\}$

Let $\mathrm{VV}=\mathrm{V}$

While notAllVerticesVisited(VV) do

Let $\mathrm{h}=$ smallestHopCount $(\mathrm{VV})$

$\mathrm{T}=\{\mathrm{v} \mid \mathrm{v} \in \mathrm{V}$ and notVisited and HopCount $(\mathrm{v})=\mathrm{h}\}$

Let improvement $=2 * \delta$

While improvement $\geq \delta$ do

For $\mathrm{t} \in \mathrm{T}$, where $\cos t\left(\mathrm{t}, \mathrm{c}_{\mathrm{t}}\right)=\max \cos \mathrm{t}$

Choose $\mathrm{c}_{\mathrm{j}} \in \mathrm{Q}$ such that $\operatorname{cost}\left(\mathrm{t}, \mathrm{c}_{\mathrm{j}}\right)=\min \cos \mathrm{t}\left(\mathrm{t}, \mathrm{c}_{\mathrm{i}}\right) \forall \mathrm{c}_{\mathrm{i}} \in \mathrm{Q}$

improvement $=\cos t\left(t, c_{t}\right)-\operatorname{cost}\left(t, c_{j}\right)$

End while

$\mathrm{VV}=\mathrm{VV}-\mathrm{T}$

End while

Fig. 3. Pseudo-code for Algorithm 1.

the MPP into two sets based on the minimization of the cost function and selects one channel for one set and the other channel for the other set (note that the MPP may have more than two children).

2) Then the algorithm examines the $h$ th hop MAPs and assigns channels to the $h$ th hop MAPs for their downlink operation. At each iteration, the $h$ th-hop MAP with the worst cost is chosen and a new channel is assigned to it to minimize the cost. This continues until the improvement of the cost function is less than a predefined threshold, denoted as $\delta$. Note that the cost function is continuously minimized at every iteration, subject to the constraint that the children of the same MAP need to use the same uplink channel. Furthermore, all $h$ th hop MAPs in the same collision domain, i.e. within $(k+1)$ hops of each other, need to be considered in the same iteration.

3) This repeats for $h=2,3, \cdots$, until each MAP is assigned a channel for upstream transmission.

4) After the algorithm assigns the available channels to the mesh nodes, it instructs the mesh nodes to switch to their assigned channels.

To avoid disturbing the operation of the entire mesh network, the channel switching procedure is performed in a bottom-up manner; it starts from the leaf nodes and stops after all nodes have switched to their designated channels.

\section{B. Channel Assignment Exploiting Traffic Load and Interfer- ence Measurements}

The second algorithm, called Algorithm 2, assigns channels based on traffic load measured at each mesh AP and on the percentage of the medium shared with external networks on all available channels. Because Algorithm 2 uses measurement results from the mesh network, it is usually invoked after the mesh network is already in operation. The goal is to minimize the following metric (termed Metric 2),

$$
\text { Minimize over } c_{j} \in C: \max \left(\sum_{i=1}^{E_{j}} \mu_{i}^{j}+\sum_{i=1}^{N_{j}} m_{i}^{j}\right),
$$

where $N_{j}$ is the number of nodes in the same collision domain that use the same channel $c_{j} \in C$ to communicate with its

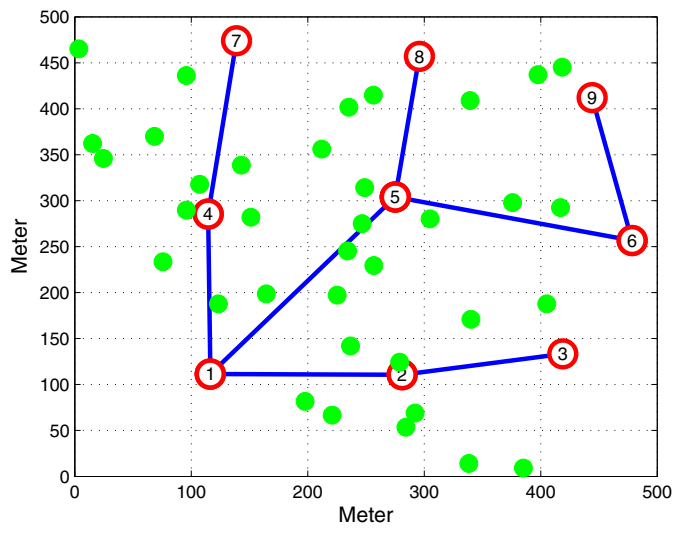

Fig. 4. A typical outdoor mesh network topology.

parent, $E_{j}$ is the number of external interferers on channel $c_{j}$, $m_{i}^{j}$ is the percentage of medium utilization of channel $c_{j}$ by the $i$ th node for transmitting packets, and $\sum_{i=1}^{E_{i}} \mu_{i}^{j}$ is the total medium utilization level of channel $c_{j}$ by external interferers. Both $m_{i}^{j}$ and $\mu_{i}^{j}$ are measured by the $i$ th node periodically according to a pre-defined measurement interval.

Similar to Algorithm 1, Algorithm 2 also adopts a centralized breadth first algorithm as the outer loop and a minmax greedy algorithm as the inner loop, where the goal is to minimize the worst cost of the $h$ th hop MAPs at every iteration. The only difference is that the objective function (2) is based on the measurement results such as traffic load and interference. In a wireless LAN deployment or a wireless mesh deployment, each AP records its own traffic load as the percentage of medium utilization on the channel. In addition, APs go off channel periodically to measure external interference on all other available channels and report the measured interference on each channel to the controller. The techniques presented in [6] can be adopted for Algorithm 2 for traffic load and interference measurement and reporting.

\section{Performance Evaluation}

To study the performance of the proposed algorithms, we implemented dual-radio mesh points and the two algorithms in ns2. We evaluated the performance of both algorithms and compare the network capacity gain of the multi-channel dualradio backhaul with a single-channel mesh backhaul.

\section{A. Simulation Setting}

Our simulation uses a typical outdoor mesh topology, as shown in Fig. 4, where nine MAPs and a number of clients are placed at random in a $500 \mathrm{~m}$ by $500 \mathrm{~m}$ area. In Fig. 4, MAP 1 is the MPP that connects to the external network. Using the Ad hoc On-demand Distance Vector (AODV) routing protocol [7], all other MAPs can compute a route to the MPP, thus resulting in a tree structure for the backhaul. The data rate on the mesh backhaul link is $18 \mathrm{Mb} / \mathrm{s}$ and the average data rate for wireless clients is $12 \mathrm{Mb} / \mathrm{s}$.

Because voice is one of the most challenging and promising applications for multi-hop outdoor mesh networks, many 


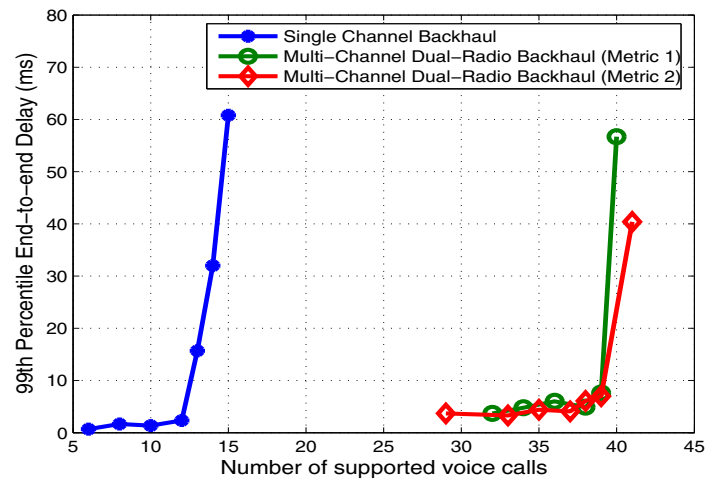

Fig. 5. End-to-end delay (single-channel backhaul vs. multi-channel dualradio backhaul) without interference from external networks.

vendors, such as BelAir, Strix, and Cisco, manufacture dualradio mesh products to support voice over mesh. Additionally, voice capacity is an essential measure of the capacity of a wireless network. In the simulations, we choose a typical voice codec, the ITU-T G.711 codec [8], which has a 160-byte voice payload and $10 \mathrm{~ms}$ codec sample interval. Each client may generate a voice call using the G.711 codec.

\section{B. Simulation Results}

In the first simulation scenario, we consider a general network setting without external IEEE 802.11 networks in the vicinity. As defined in IEEE 802.11e, the delay budget for a voice call over a wireless LAN is set to $10 \mathrm{~ms}$. Moreover, the acceptable packet loss rate for the G.711 codec is $1 \%$.

We compare the voice capacity of a single channel mesh backhaul with that of a multi-channel dual-radio backhaul. As shown in Fig. 5, the $x$-axis represents the number of supported voice calls and the $y$-axis represents the 99th percentile of the voice packet delay. ${ }^{2}$ Note that the 99 th percentile delay (not the average delay) is chosen here because any voice packet that experiences delay more than $10 \mathrm{~ms}$ may be discarded. For acceptable voice quality, the packet drop rate has to be less than $1 \%$, which means $99 \%$ of the packets should have delays smaller than $10 \mathrm{~ms}$.

Figure 5 shows that about 12 G.711 VoIP calls can be supported simultaneously over a single-channel mesh backhaul. But the multi-channel dual-radio backhaul can support 39 G.711 VoIP calls, offering more than a $300 \%$ capacity improvement. When there is no external interference for the selected network scenario, both channel selection algorithms yield similar results.

Another interesting scenario is that once the system accepts more voice calls than it can support, the end-to-end delay increases dramatically. This is because after the mesh backhaul reaches its voice capacity, the queues at each MAP start to grow quickly and eventually overflow.

Figure 6 shows that when a single-channel backhaul supports 11 or fewer voice calls, the packet loss rate is less than

\footnotetext{
${ }^{2}$ The 99th percentile delay means $99 \%$ of the packets experience smaller delays than the indicated value.
}

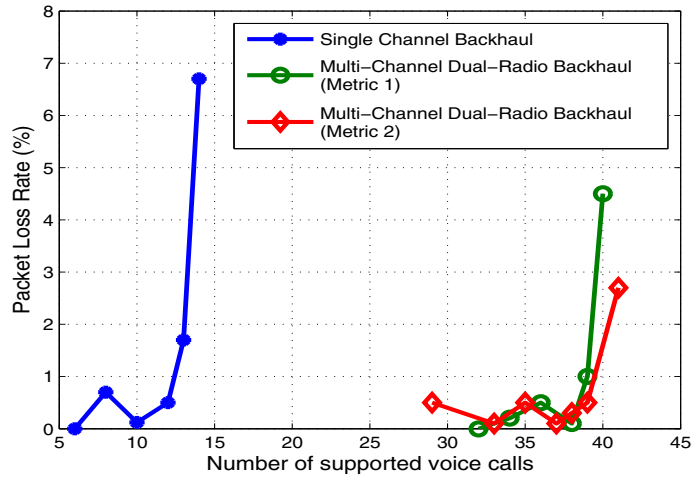

Fig. 6. Packet loss rate (single-channel backhaul vs. multi-channel dual-radio backhaul)without interference from external networks.

1\%. A multi-channel dual radio backhaul can support up to 39 voice calls. In this case, the rapid increase in packet loss rate after the network voice capacity is reached is also caused by queue overflows.

For the second simulation scenario, we consider a general network topology with an external 802.11 network in the vicinity of the MPP. We assume the external 802.11 network takes up 50\% medium time on Channel 1, which is one of the two channels utilized by MPP.

We compare the performance of the algorithm using Metric 1 (see Eqn. (1)) and Metric 2 (see Eqn. (2)). Because Metric 1 only considers the traffic load on each MP within the mesh network, the bandwidth taken by the external 802.11 network is not considered. In comparison, Metric 2 considers both external interference and the traffic load within the mesh network. As shown in Figs. 7 and 8, Algorithm 2 supports about eight additional VoIP calls than Algorithm 1, which offers $27 \%$ higher network capacity in this case.

\section{RELATED WORK}

A significant body of prior work examines the benefits of utilizing multiple channels for wireless mesh networks. We summarize them into two categories: i) modifying the 802.11 MAC protocol to support multiple channels, and ii) adopting multiple radios to support multiple channels.

References [9]-[11] belong to the first category. The key advantage of such schemes is that only a single radio is needed to support multiple channels, which lowers the hardware cost. However, these schemes require changes to the 802.11 MAC protocol and they are not backward compatible. Furthermore, they require per packet channel switching, which demands a channel switch delay that is one or two magnitude lower than what can be supported by today's hardware.

References [3], [4], [12], [13] and [5] fall into the second category. References [3], [4] and [12] utilize two or more radios for capacity improvement in an ad hoc network setting. Gong et al. assume that all nodes are equipped with two radios and they share a common control channel to exchange channel assignment information [3]. Data channels are assigned on a per transmitter basis. Because all nodes share a common 


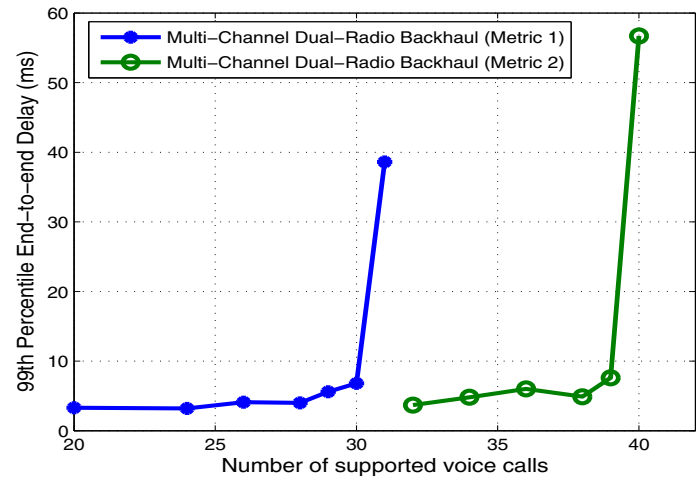

Fig. 7. End-to-end delay (Algorithm 1 vs. Algorithm 2) with interference from external networks.

control channel, service disruption is kept to a minimum when a transceiver switches from one channel to another. Kyasanure et al. propose to statically assign channels to a subset of radios on each node [12]. The remaining radios dynamically switch to the static channels assigned to neighboring nodes to communicate with them. References. [3], [4] and [12] require that radios switch between channels on a per packet basis, which poses challenges for hardware design.

Raniwala et al. propose a centralized load-aware algorithm to dynamically assign channels in a multi-radio mesh network [13]. Through a joint design of the routing and channel assignment algorithm, a factor of three or eight improvement can be achieved when compared with a single backhaul radio single channel mesh network. However, because route changes may cause packet losses and packet reordering at intermediate routers, TCP performance can be negatively affected.

The work by Ramachandra et al. [5] shares similar design philosophies as the proposed algorithm. First, channel assignment is separated from the routing algorithm. Secondly, channels are selected to minimize both interference within the mesh network and between the mesh network and colocated wireless networks. The important differences between this work and [5] are i) [5] assumes all nodes share a common control channel, whereas we do not make such an assumption, and ii) [5] estimates interference based on the number of co-located interfering radios, whereas our work considers measurements in terms of percentage of medium utilization by interfering radios, which is generally more accurate.

\section{CONCLUSION}

We have considered the channel assignment problem for multi-channel dual-radio mesh backhauls. Two channel assignment algorithms are proposed, which are complementary of each other. Without relying on measurement results, Algorithm 1 seeks to balance the mesh traffic load over any channel at initial network set up. Once the network is in operation and the measurement results become available, Algorithm 2 considers both measured mesh traffic load and interference from external networks. Through ns2 simulations with voice sessions, we observe over three fold improvement in the network capacity.

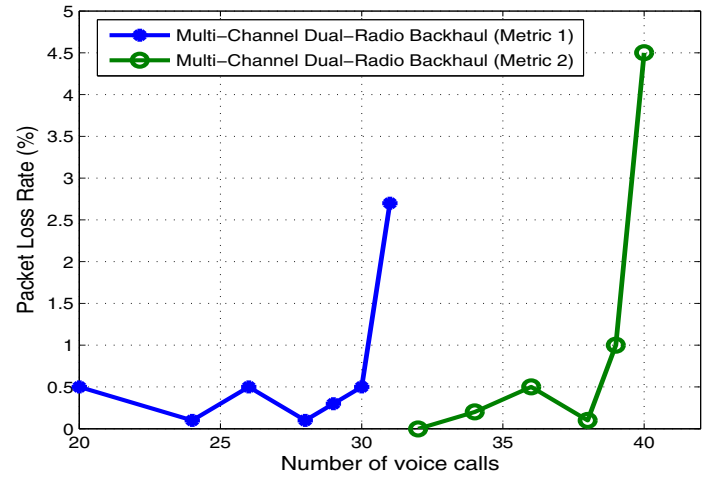

Fig. 8. Packet loss rate (Algorithm 1 vs. Algorithm 2) with interference from external networks.

Moreover, simulation results show that considering both external interference and mesh traffic load, the network capacity can be further improved by up to $27 \%$.

\section{ACKNOWLEDGMENT}

$\mathrm{S}$. Mao's research is supported in part by the National Science Foundation (NSF) under grant ECCS-0802113 and through the Wireless Internet Center for Advanced Technology (WICAT) at Auburn University. This work was also supported in part by the NSF, while S. Midkiff was working at the Foundation. Any opinion, finding, and conclusions or recommendations expressed in this material are those of the authors and do not necessarily reflect the views of the NSF.

\section{REFERENCES}

[1] P. Gupta and P. R. Kumar, "The capacity of wireless networks," IEEE Trans. Information Theory, vol. 48, no. 2, pp. 388-404, Mar. 2000.

[2] J. Li, et al., "Capacity of ad hoc networks," in Proc. ACM MobiCom'01, Rome, Italy, July 2001, pp. 61-69.

[3] M. Gong, S. Midkiff, and S. Mao, "A cross-layer approach to channel assignment in wireless ad hoc networks," ACM/Springer Journal of Mobile Networks and Applications, vol. 12, no. 1, pp. 43-56, Feb. 2007.

[4] — "On-demand routing and channel assignment in multi-channel mobile ad hoc networks," Ad Hoc Networks Journal, in press.

[5] K. Ramachandran, et al., "Interference-aware channel assignment in multi-radio wireless mesh networks," in Proc. IEEE INFOCOM'06, Barcelona, Spain, Apr. 2006, pp. 1-12.

[6] I. L. S. Committee, "Part 11 amendment 1: Radio resource measurement of wireless lan's," in IEEE $p 802.11 \mathrm{k}, 2008$.

[7] C. Perkins, E. Belding-Royer, and S. Das, "Ad hoc on-demand distance vecor (AODV) routing," July 2003, IETF RFC 3561.

[8] I. R. G.711, "Pulse code modulation (pcm) of voice frequencies," Dec. 1988.

[9] P. Bahl, R. Chandra, and J. Dunagan, "SSCH: Slotted seeded channel hopping for capacity improvement in IEEE 802.11 ad hoc wireless networks," in Proc. ACM MobiCom'04, Philadelphia, PA, Sept. 2004, pp. 216-230.

[10] N. Jain, S. Das, and A. Nasipuri, "A multichannel CSMA MAC protocol with receiver-based channel selection for multihop wireless networks," in Proc. IEEE ICCCN'01, Scottsdale, AZ, Oct. 2001, pp. 432-439.

[11] J. So and N. Vaidya, "Multi-channel MAC for ad hoc networks: Handling multi-channel hidden terminals using a sigle transceiver," in Proc. ACM MobiHoc'04, Tokyo, Japan, May 2004, pp. 222-233.

[12] P. Kyasanur and N. Vaidya, "Routing and interface assignment in multichannel multi-interface wireless networks," in Proc. IEEE WCNC'05, New Orleans, LA, Mar. 2005, pp. 2051-2056.

[13] A. Raniwala and T. Chiueh, "Architecture and algorithms for an IEEE 802.11-based multi-channel wireless mesh network," in Proc. IEEE INFOCOM’05, Miami, FL, Mar. 2005, pp. 2223-2234. 